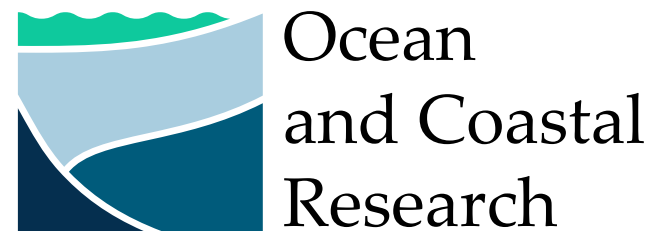

\title{
Phytoplankton community in a tropical estuarine gradient after an exceptional harmful bloom of Akashiwo sanguinea (Dinophyceae) in the Todos os Santos Bay
}

\author{
Helen Michelle de Jesus Affe ${ }^{1,2, *}$, Lorena Pedreira Conceição ${ }^{3,4}$, Diogo Souza Bezerra Rocha ${ }^{5}$, Luis Antônio de \\ Oliveira Proença ${ }^{6}$, José Marcos de Castro Nunes ${ }^{3,4}$ \\ 1 Universidade do Estado do Rio de Janeiro - Faculdade de Oceanografia (Bloco E - 900, Pavilhão João Lyra Filho, $4^{\circ}$ andar, sala 4018, \\ R. São Francisco Xavier, 524 - Maracanã - 20550-000 - Rio de Janeiro - RJ - Brazil) \\ ${ }^{2}$ Instituto Nacional de Pesquisas Espaciais/INPE - Rede Clima - Sub-rede Oceanos (Av. dos Astronautas, 1758. Jd. da Granja -12227-010 - \\ São José dos Campos - SP - Brazil) \\ ${ }^{3}$ Universidade Estadual de Feira de Santana - Departamento de Ciências Biológicas - Programa de Pós-graduação em Botânica \\ (Av. Transnordestina s/n - Novo Horizonte - 44036-900 - Feira de Santana - BA - Brazil) \\ ${ }^{4}$ Universidade Federal da Bahia - Instituto de Biologia - Laboratório de Algas Marinhas (Rua Barão de Jeremoabo, 668 - Campus de \\ Ondina 40170-115 - Salvador - BA - Brazil) \\ ${ }^{5}$ Instituto Internacional para Sustentabilidade - (Estr. Dona Castorina, 124 - Jardim Botânico - 22460-320 - Rio de Janeiro - RJ - Brazil) \\ ${ }^{6}$ Instituto Federal de Santa Catarina (Av. Ver. Abrahão João Francisco, 3899 - Ressacada, Itajaí - 88307-303 - SC - Brazil) \\ * Corresponding author: helenmaffe@gmail.com
}

\section{ABSTRACT}

The objective of this study was to evaluate variations in the composition and abundance of the phytoplankton community after an exceptional harmful bloom of Akashiwo sanguinea that occurred in Todos os Santos Bay (BTS) in early March, 2007. Samples were collected every ten days, between April, 2007 and March, 2008, from the estuarine gradient of the Paraguaçu River to BTS. The physical and chemical variables were measured in situ using a multiparameter sensor. Water samples were collected for analysis of the dissolved inorganic nutrient concentrations and for the study of composition and abundance of the microphytoplankton. Overall, 135 taxa were identified, with a higher richness of diatoms. The total cell density ranged from $2.92 \times 10^{3}$ to $1.16 \times 10^{7}$ $\left(5.47 \times 10^{5} \pm 1.69 \times 10^{6}\right)$ cells $\mathrm{L}^{-1}$, with higher values in the freshwater zone than in the marine area. Five species showed peaks of abundance throughout the study, forming small blooms. Four of these blooms occurred in the rainy season, formed by the species Guinardia striata (April, 2007), Scrippsiella cf. acuminata (August, 2007), Euglena gracilis (August, 2007), and Skeletonema cf. costatum (September, 2007), while a new bloom of the species Akashiwo sanguinea occurred during the dry season (December, 2007). The environment was typically oligotrophic, with low spatiotemporal variation in the concentrations of dissolved nutrients. Even so, we observed short-term variations in the structure and composition of the phytoplankton community, demonstrated by rapid bloom events, followed by an increase in the total abundance of microphytoplankton, especially during the rainy season. The blooms did not cause any notable changes in the water column and did not present any harmful effects on the system.

Descriptors: Algal blooms, Brazilian coast, Marine phytoplankton, Paraguaçu river estuary.

\section{INTRODUCTION}

The composition and dynamics of phytoplankton communities in estuarine systems are modulated by

Submitted on: 21-August-2020

Approved on: 19-January-2021

Editor: Rubens M. Lopes

(C) 2021 The authors. This is an open access article distributed under the terms of the Creative Commons license. the variability of abiotic and ecological factors such as gradients of salinity, nutrients, temperature, and inter- and intraspecific interactions (Rabalais, 2002; Cloern and Dufford, 2005; Litchman and Klausmeier, 2008; Córdoba-Mena et al., 2020). Algae are of significant ecological importance as indicators of ecosystem changes. The fluctuation of physical and chemical variables under the influence of tidal cycles and rainfall events are reflected very rapidly by 
phytoplankton, presenting changes in composition, diversity, and abundance of the community, thereby affecting ecosystem function (Morse et al., 2014; Moser et al., 2017; Affe et al., 2018).

Qualitative and quantitative changes in phytoplankton community and primary productivity in tropical environments are usually related to the increase in the input of dissolved nutrients in the system (Heisler et al., 2008). These changes are often related to the formation of blooms, which are usually natural events, corresponding to a significant increase in biomass in the organic production chain (Smayda, 1997). On the other hand, blooms of harmful species can harm ecosystem services by compromising water quality, aesthetic characteristics, and recreational activities, for example, and such blooms appear to be increasing in frequency and magnitude in different systems (Moore et al., 2008; Hallegraeff, 2010; Affe et al., 2016).

Because estuarine systems are highly hydrodynamic, the trigger responsible for iniciating a bloom has yet to be identified, and short-term monitoring is required to identify rapid changes in water quality and algal biomass (Morse et al., 2014). In general, the bloom is detected only when it becomes visible, covering the water surface (Egerton et al., 2014) or due to its deleterious effects, such as the harmful algal bloom of the species Akashiwo sanguinea (Hirasaka) G. Hansen and Moestrup, which occurred in early March, 2007 in Todos os Santos Bay (BTS). This bloom caused the death of more than 50 tons of fish due to anoxic conditions in the water column and obstruction of the gills caused by the rapid increase in the biomass of this dinoflagellate (Reis-Filho et al., 2012).

This bloom event revealed an important knowledge gap about phytoplankton in the Paraguaçu River estuary, which had persisted until then, although this is one of the main coastal aquatic systems of Northeastern Brazil. Thus, the present study aimed to analyze short-term variations in the composition and abundance of the phytoplankton community after the exceptional harmful bloom of Akashiwo sanguinea, as the first study on the dynamics of the community along this estuarine gradient.

\section{METHODS}

\section{STUdY AREA}

The Paraguaçu River estuarine system $\left(12^{\circ} 00^{\prime} \mathrm{S}\right.$ and $13^{\circ} \mathrm{S}$ and $38^{\circ} 30^{\prime} \mathrm{W}$ and $39^{\circ} 30^{\prime} \mathrm{W}$ ) (Figure 1) is approximately $80 \mathrm{~km}^{2}$ in area, with a volume of about $4,650,000$ $\mathrm{m}^{3}$ (Genz et al., 2008). The climate of the region is hot and humid, with an average annual temperature of $24^{\circ} \mathrm{C}$ (Genz et al., 2008). Throughout its course, the estuarine system is affected by anthropogenic activities, which involve the artificial control of the hydrological regime by the Pedra do Cavalo dam, domestic effluent discharges, agriculture, and subsistence activities of the human populations, based on consumption and commercialization of fish and shellfish (Hydros, 2005; Lessa et al., 2001).

The estuarine system is divided between the low river course (freshwater zone), the Iguape Bay (transition zone), and the Paraguaçu channel, flowing into BTS $\left(12^{\circ} 50 \mathrm{~S} 38^{\circ} 38 \mathrm{~W}\right)$ as the main tributary of the same (Genz et al., 2008). The BTS is about $1223 \mathrm{~km}^{2}$ in length and has an average depth of $9.8 \mathrm{~m}$ (Cirano and Lessa, 2007). Internal circulation in the bay is forced by semi-diurnal tides, which, in addition to the seasonal pattern of wind circulation, the main forcing factor in the surface layers, conditions a well-mixed water column with oceanic characteristics (Lessa et al., 2009; Santana et al., 2018).

\section{SAMPLING AND ANALYSES}

Six stations along the course of the estuarine system (Figure 1) were sampled every ten days, over twelve months (April, 2007 to March, 2008). The physical and chemical variables (i.e., temperature, salinity, dissolved oxygen, and $\mathrm{pH}$ ) were measured in situ, in the subsurface ( $0.5 \mathrm{~m}$ depth), using a multiparameter sensor. To analyze the dissolved inorganic nutrients (i.e., nitrite, nitrate, and phosphate), $1 \mathrm{~L}$ water samples were collected at each point using a Van Dorn bottle. The samples were immediately filtered after each collection through fiberglass filters (Whatman GF/F - $0.7 \mu \mathrm{m}$ pore), using a vacuum pump. Aliquots of $250 \mathrm{~mL}$ of the filtrated volume of each sample were kept frozen until the respective analyses were carried out by the classic colorimetric method, adapted from Strickland and Parsons (1972). The rainfall data were obtained by consulting the database from the Weather Forecast and Climate Studies Center (https://www.cptec.inpe.br/). 

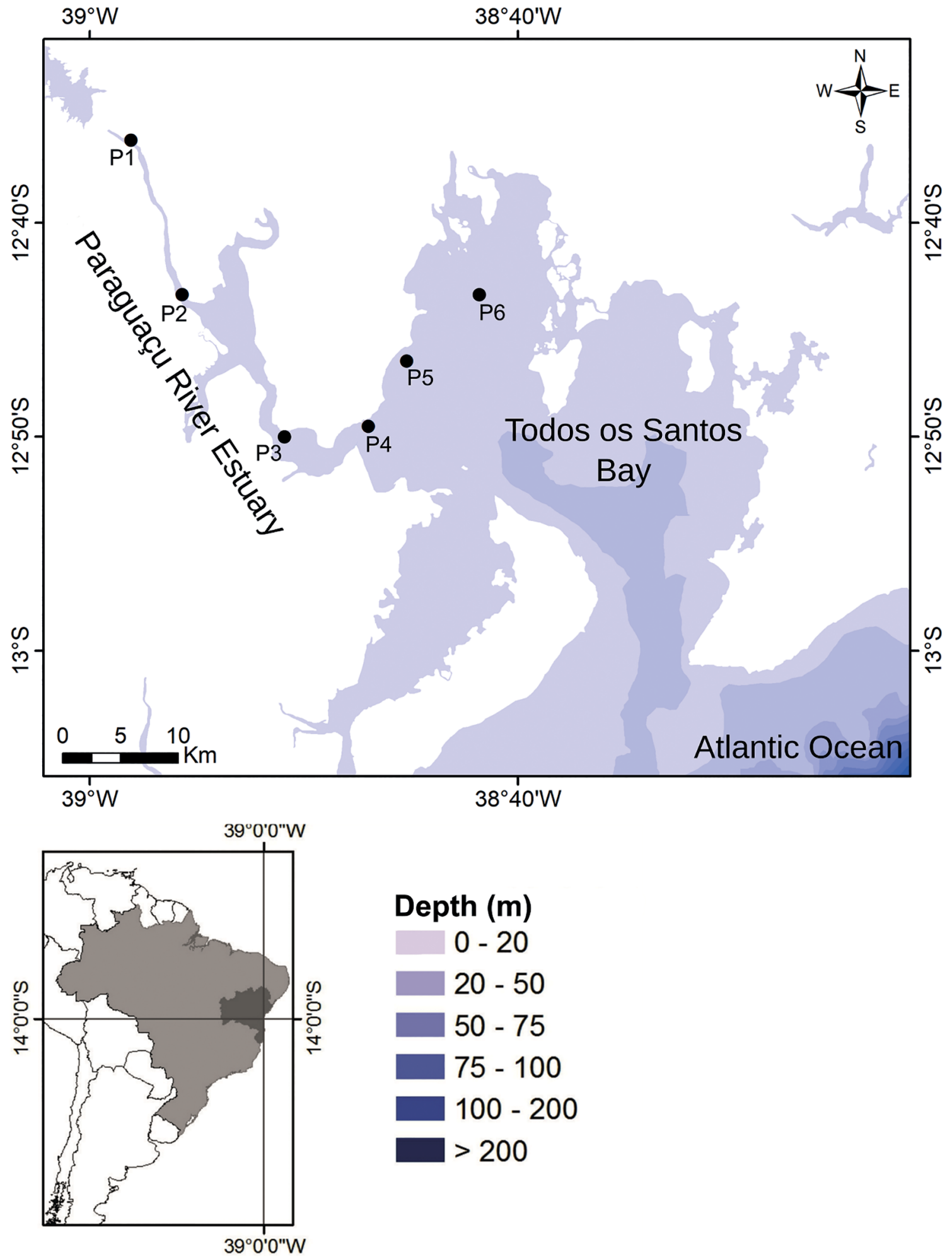

Figure 1. Paraguaçu estuarine gradient - Todos os Santos Bay, with the locations of sampling stations in the freshwater zone (P1), transitional zone (P2, P3, and P4) and marine zone (P5 and P6). ${ }^{*}$ The distribution of points was defined to cover the entire estuarine gradient in the sample design. The delimitation of the estuarine zones was defined posteriorly, based on an analysis of salinity (refer to Results section). 
For phytoplankton analyses, water samples ( 250 $\mathrm{mL}$ at each collection point) were collected using a plankton net $(20 \mu \mathrm{m})$ in horizontal tows. Subsurface ( $0.5 \mathrm{~m}$ depth) water samples (1L) were collected at each station using a Van Dorn bottle. Each water sample was stored in dark bottles and fixed with $1 \%$ lugol. In the laboratory, samples were analyzed on slides under a light microscope (Olympus CX31), with 200x or 400x magnification, to identify the microphytoplankton taxa. Samples were analyzed according to the Utermöhl method (1958), counting the entire bottom of the chamber ( 50 or $100 \mathrm{~mL}$ ), under an inverted microscope (Olympus IX50) to determine microphytoplankton cell densities (cell $\mathrm{L}^{-1}$ ).

To characterize the microphytoplankton composition and structure along the estuarine gradient, species richness $(\mathrm{S})$, Shannon diversity index $\left(\mathrm{H}^{\prime}\right)$, Pielou's uniformity index $\left(J^{\prime}\right)$, and abundance (cell density) were estimated. The data normality of the community abundance and environmental variables was verified using the Kolmogorov-Smirnov test; homogeneity of variance was tested using Levene's test. The Kruskal-Wallis analysis of variance, followed by the $p$-values multiple comparison test, was used to assess the occurrence of significant differences ( $p<$ 0.05 ) in the community abundance, as well as the differences in abiotic variables in the estuarine gradient, between the dry and rainy seasons.

Spearman's correlation tests were performed to analyze possible correlations of rainfall with salinity and of these factors with the abundance of the phytoplankton community in the estuarine gradient. Non-metric multidimensional scaling (NMDS) analysis was carried out based on distance matrices, calculated from the Bray-Curtis index, to observe the ranking of the samples as functions of the dissimilarity in species composition and abundance along the estuarine gradient, between the dry and rainy seasons. Variance partition analysis (Borcard et al., 1992; Peres-Neto et al., 2006; Oksanen et al., 2019) was used to verify the relative contribution of physical and chemical variables, dissolved inorganic nutrients, and rain in the microphytoplankton abundance along the estuarine gradient, in the dry and rainy seasons. All statistical analyses were performed using $R$ statistical software (R Core Team, 2020).

\section{RESULTS}

\section{Abiotic Variables}

Based on the variations in rainfall (Figure 2), we characterized rainy season as the months with average precipitation greater than $100 \mathrm{~mm}$, with a monthly average of $151.18( \pm 44.43) \mathrm{mm}$, while the monthly average in the dry period was $44.33( \pm 31.68) \mathrm{mm}$. The estuarine gradient was characterized by a wide variation in salinity (Figure 2), based on which, three estuarine zones were characterized, with significant variation in salinity in both pluviometric seasons $(p$ < 0.001): (1) freshwater zone, represented by P1 site; (2) transitional zone, represented by P2 to P4 sites; and (3) marine zone represented by P5 and P6 sites (Figure 1). We observed that in the freshwater zone, there was a clear pattern of variation in salinity as a function of rainfall (Figure 2). The Spearman test showed a negative correlation between these variables $(\rho=-0.68)$ in this zone, with lower correlation in the transition $(\rho=-0.23)$ and marine $(\rho=-0.46)$ zones.

The other physical and chemical variables analyzed did not show significant variations along the estuarine gradient (between the zones) or between the dry and rainy seasons $(p>0.05)$. The water temperature presented a low average monthly variation, with the greatest amplitude of variation $\left(7^{\circ} \mathrm{C}\right)$ occurring in the transition zone between the dry and rainy seasons. The concentrations of dissolved oxygen (OD) were occasionally below $6 \mathrm{mg} \mathrm{L}^{-1}$ (with $>6$ being the reference value for estuaries), and the $\mathrm{pH}$ values showed the typical alkalinity characteristic of estuarine waters (i. e., $>7$ and $<8.5$ ) (Table 1).

The dissolved inorganic nutrients showed concentration patterns that were characteristic of oligotrophic environments, along the estuarine gradient. Nitrite was the nutrient with the greatest variation between the two rainfall stations (Table 1), with concentrations below the detection limit of the method, at all sampling points in January (dry season). In the rainy season, significant increases in nitrite concentration were recorded in the three estuarine zones ( $F Z, p=0.023 ; \mathrm{TZ}, p=0.027 ; \mathrm{MZ}, p=0.024)$. For nitrate, the lowest concentrations were always recorded in the marine zone (Table 1), but without significant differences $(p>0.05)$ with the other estuarine zones, or 


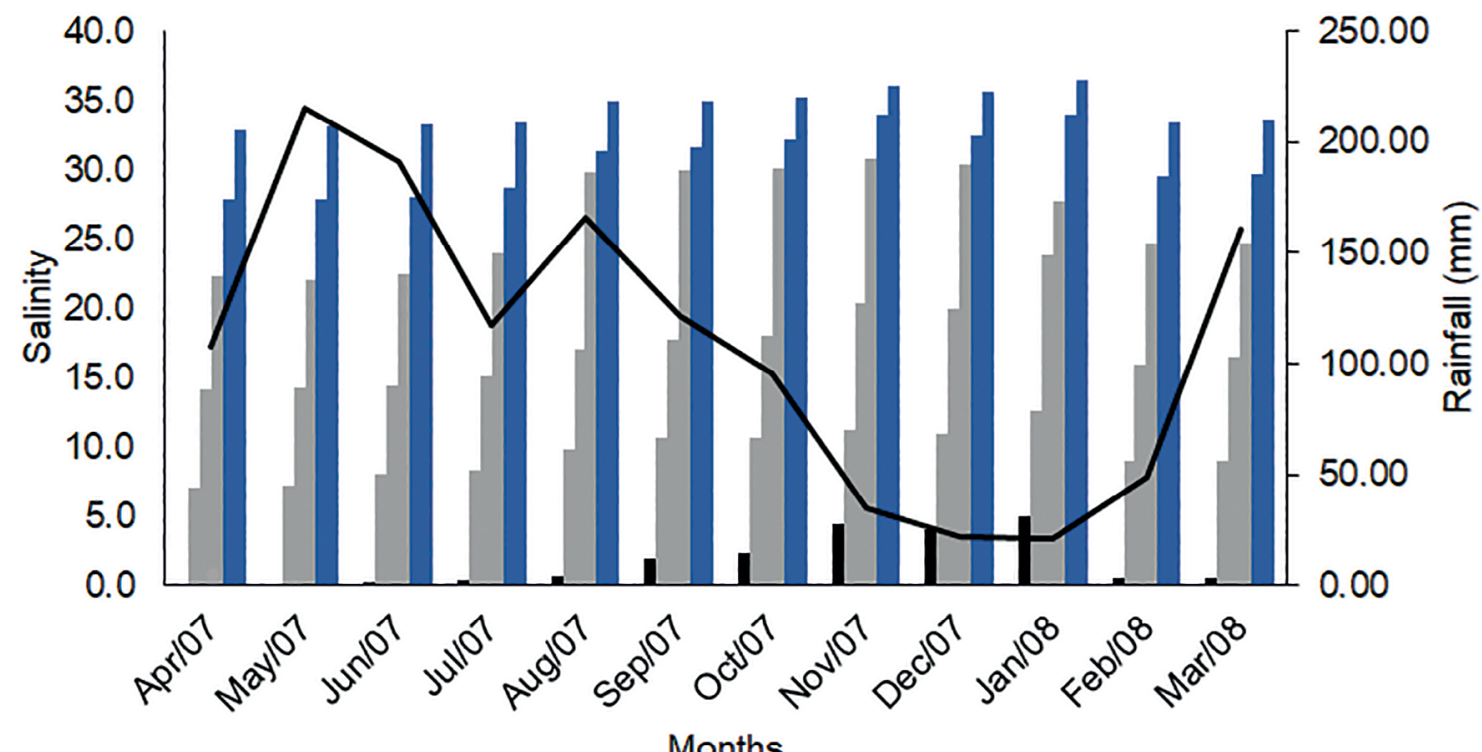

m $=\mathrm{TZ}=\mathrm{MZ}$-Rainfall

Figure 2. Monthly rainfall variability (line), and monthly and spatial salinity variability (bars) between April, 2007 and March, 2008 in the Paraguaçu estuarine gradient. $\mathrm{FZ}=$ freshwater zone; $\mathrm{TZ}$ = transitional zone; $\mathrm{MZ}=$ marine zone.

between the dry and rainy seasons. The same pattern of low spatio-temporal variation was recorded for phosphate (Table 1).

\section{StRUCTURE AND COMPOSITION OF THE PHYTOPLANKTON COMMUNITY}

A total of 135 taxa were identified (Table 2). Diatoms were the most representative group $(60 \%$ of taxa), followed by dinoflagellates $(16 \%)$, cyanobacteria (9\%), chlorophytes (5\%), desmids (5\%), and euglenophytes (5\%). The transition zone was the area with the highest species richness, both in the dry and rainy seasons, always followed by the marine zone. The species diversity index varied between 2.91 to 4.05 bits ind $^{-1}$, with an increase in the average diversity along the estuarine gradient, highest in the marine zone, as well as having a discrete increase during the dry season in the three estuarine zones (Figure 3 ). The same pattern was also registered for the evenness index; there was no dominance of any taxon ( $J$ ' ranged between 0.82 and 0.96 ) in the estuarine zones in any of the pluviometric stations.

The total phytoplankton density varied between $2.92 \times 10^{3}$ and $1.16 \times 10^{7}\left(5.47 \times 10^{5} \pm 1.69 \times 10^{6}\right)$ cells $\mathrm{L}^{-1}$. The transitional zone showed the highest average densities in both the dry and rainy seasons, except in June and July (Figure 3). Blooms of five species were identified during the study period. In the rainy season, Guinardia striata (Stolterfoth) Hasle $\left(1.39 \times 10^{6}\right.$ cell $\left.\mathrm{L}^{-1}\right)$, Euglena gracilis G.A.Klebs $\left(2.71 \times 10^{6} \mathrm{cell} \mathrm{L}^{-1}\right)$, and Skeletonema cf. costatum (Greville) Cleve (1.16 $\times$ $\left.10^{6} \mathrm{cell} \mathrm{L}^{-1}\right)$, formed blooms in the transitional zone in the months of April, August and September, 2007, respectively, and Scrippsiella cf. acuminata (Ehrenberg) Kretschmann, Elbrächter, Zinssmeister, S.Soehner, Kirsch, Kusber \& Gottschling $\left(7.23 \times 10^{6}\right.$ cell L-1 $\left.^{-1}\right)$ formed a bloom (August, 2007) in the freshwater zone. In the dry season (December, 2007), a bloom of Akashiwo sanguinea (K. Hirasaka) Gert Hansen \& Moestrup $\left(1.03 \times 10^{6}\right.$ cells $\left.\mathrm{L}^{-1}\right)$ occurred in the transitional zone (Figure 3). Unlike the exceptional harmful bloom of this species recorded in 2007, which covered an extensive area in BTS, this new bloom occurred in a punctual area, restricted to the $\mathrm{P} 3$ site. All five blooms recorded showed a fast development cycle ( $<10$ days), and were not detected in the collections made immediately after their registries. Because they were relatively small blooms, no changes in water characteristics that could characterize potential harmful effects were detected. 
Table 1. Minimum - maximum (average \pm standard deviation) of environmental variables: salinity, temperature $\left({ }^{\circ} \mathrm{C}\right)$, dissolved oxygen $\left(\mathrm{mg} \mathrm{L}^{-1}\right), \mathrm{pH}$, concentration of dissolved inorganic nutrients: nitrite, nitrate, and phosphate ( $\left.\mu \mathrm{M}\right)$ in the rainy (April-September, 2007, and March, 2008) and dry (October-December, 2007, and January and February, 2008) seasons in the Paraguaçu estuarine gradient. $\mathrm{FZ}=$ freshwater zone; $\mathrm{TZ}=$ transitional zone; $\mathrm{MZ}=\mathrm{marine} z$ zone.

\begin{tabular}{|c|c|c|c|c|c|c|c|c|c|}
\hline Season & Month & Zone & Salinity & Temperature & $\begin{array}{c}\text { Dissolved } \\
\text { oxygen }\end{array}$ & pH & Nitrite & Nitrate & Phosphate \\
\hline \multirow[t]{18}{*}{ Rainy } & April & $\mathbf{F Z}$ & $\begin{array}{c}0-0.20 \\
(0.13 \pm 0.12)\end{array}$ & $\begin{array}{c}27.0-27.4 \\
(27.2 \pm 0.21)\end{array}$ & $\begin{array}{c}6.6-6.7 \\
(6.6 \pm 0.02)\end{array}$ & $\begin{array}{c}7.9-8.0 \\
(7.9 \pm 0.01)\end{array}$ & $\begin{array}{c}0.60-0.70 \\
(0.67 \pm 0.06)\end{array}$ & 0.05 & $\begin{array}{c}0.03-0.38 \\
(0.11 \pm 0.14)\end{array}$ \\
\hline & & TZ & $\begin{array}{c}7.0-22.5 \\
(14.5 \pm 6.6) \\
\end{array}$ & $\begin{array}{c}26.7-27.4 \\
(26.9 \pm 0.21)\end{array}$ & $\begin{array}{c}5.2-6.9 \\
(6.2 \pm 0.79) \\
\end{array}$ & $\begin{array}{c}7.9-8.0 \\
(7.9 \pm 0.02) \\
\end{array}$ & $\begin{array}{c}0.40-0.70 \\
(0.60 \pm 0.11)\end{array}$ & $\begin{array}{c}0.04-0.09 \\
(0.07 \pm 0.02)\end{array}$ & $\begin{array}{c}0.03-0.09 \\
(0.09 \pm 0.09) \\
\end{array}$ \\
\hline & & MZ & $\begin{array}{c}27.8-32.9 \\
(30.3 \pm 2.76)\end{array}$ & $\begin{array}{c}27.6-27.9 \\
(27.7 \pm 0.10)\end{array}$ & $\begin{array}{c}6.0-6.2 \\
(6.1 \pm 0.12)\end{array}$ & $\begin{array}{c}7.8-7.9 \\
(7.9 \pm 0.03)\end{array}$ & $\begin{array}{c}0.30-1.20 \\
(0.78 \pm 0.46)\end{array}$ & $\begin{array}{c}0.05-0.09 \\
(0.08 \pm 0.02)\end{array}$ & 0.03 \\
\hline & \multirow[t]{3}{*}{ May } & $\mathbf{F Z}$ & $\begin{array}{c}0-0.20 \\
(0.13 \pm 0.12)\end{array}$ & $\begin{array}{c}27.6-27.7 \\
(27.6 \pm 0.06)\end{array}$ & $\begin{array}{c}5.0-5.2 \\
(5.1 \pm 0.10)\end{array}$ & $\begin{array}{c}7.4-7.5 \\
(7.5 \pm 0.01)\end{array}$ & 1.00 & 0.05 & 0.13 \\
\hline & & $\mathbf{T Z}$ & $\begin{array}{c}7.1-22.2 \\
(14.5 \pm 6.47)\end{array}$ & $\begin{array}{c}27.5-29.5 \\
(28.3 \pm 0.76)\end{array}$ & $\begin{array}{c}5.0-5.7 \\
(5.3 \pm 0.31)\end{array}$ & $\begin{array}{c}7.1-7.5 \\
(7.3 \pm 0.15)\end{array}$ & $\begin{array}{c}0.30-0.70 \\
(0.53 \pm 0.14)\end{array}$ & $\begin{array}{c}0.05-0.09 \\
(0.07 \pm 0.02)\end{array}$ & $\begin{array}{c}0.10-0.16 \\
(0.13 \pm 0.02)\end{array}$ \\
\hline & & MZ & $\begin{array}{c}27.7-33.2 \\
(30.5 \pm 2.92)\end{array}$ & $\begin{array}{c}27.8-28.4 \\
(28.1 \pm 0.25)\end{array}$ & $\begin{array}{c}6.2-6.6 \\
(6.4 \pm 0.22)\end{array}$ & $\begin{array}{c}7.7-7.9 \\
(7.8 \pm 0.12)\end{array}$ & $\begin{array}{c}0.30-0.60 \\
(0.47 \pm 0.12)\end{array}$ & $\begin{array}{c}0.07-0.09 \\
(0.08 \pm 0.01)\end{array}$ & $\begin{array}{c}0.06-0.09 \\
(0.08 \pm 0.02)\end{array}$ \\
\hline & \multirow[t]{3}{*}{ June } & $\mathbf{F Z}$ & $\begin{array}{c}0.30-0.40 \\
(0.33 \pm 0.06)\end{array}$ & $\begin{array}{c}27.7-27.9 \\
(27.8 \pm 0.10)\end{array}$ & 7.80 & 8.00 & 0.70 & 0.05 & 0.03 \\
\hline & & $\mathbf{T Z}$ & $\begin{array}{c}8.0-22.5 \\
(14.9 \pm 6.26)\end{array}$ & $\begin{array}{c}27.4-28.3 \\
(27.7 \pm 0.34)\end{array}$ & $\begin{array}{c}6.3-7.9 \\
(6.9 \pm 0.69) \\
\end{array}$ & $\begin{array}{c}7.9-8.0 \\
(8.0 \pm 0.02) \\
\end{array}$ & $\begin{array}{c}0.30-0.70 \\
(0.58 \pm 0.16)\end{array}$ & $\begin{array}{c}0.05-0.09 \\
(0.07 \pm 0.02)\end{array}$ & 0.03 \\
\hline & & MZ & $\begin{array}{c}28-33.3 \\
(30.6 \pm 2.85)\end{array}$ & $\begin{array}{c}27.0-27.5 \\
(27.2 \pm 0.23)\end{array}$ & $\begin{array}{c}7.2-7.3 \\
(7.2 \pm 0.05)\end{array}$ & 8.00 & $\begin{array}{c}0.40-1.20 \\
(0.78 \pm 0.42)\end{array}$ & $\begin{array}{c}0.07-0.09 \\
(0.08 \pm 0.01)\end{array}$ & $\begin{array}{c}0.02-0.03 \\
(0.03 \pm 0.01)\end{array}$ \\
\hline & \multirow[t]{3}{*}{ July } & $\mathbf{F Z}$ & 0.40 & $\begin{array}{c}27.5-27.6 \\
(27.5 \pm 0.06)\end{array}$ & 8.20 & 8.00 & 0.50 & 0.05 & 0.03 \\
\hline & & TZ & $\begin{array}{c}8.2-24 \\
(15.8 \pm 6.83) \\
\end{array}$ & $\begin{array}{c}25-27.1 \\
(26.2 \pm 0.92)\end{array}$ & $\begin{array}{c}7.2-8.1 \\
(7.6 \pm 0.42)\end{array}$ & $\begin{array}{c}7.0-8.0 \\
(7.4 \pm 0.48) \\
\end{array}$ & $\begin{array}{c}0.30-0.50 \\
(0.43 \pm 0.10)\end{array}$ & $\begin{array}{c}0.05-0.09 \\
(0.07 \pm 0.02)\end{array}$ & $\begin{array}{c}0.03-0.08 \\
(0.04 \pm 0.03) \\
\end{array}$ \\
\hline & & MZ & $\begin{array}{c}28-33.5 \\
(31.0 \pm 2.62)\end{array}$ & $\begin{array}{c}25.8-26.6 \\
(26.2 \pm 0.43)\end{array}$ & $\begin{array}{c}6.8-7.6 \\
(7.2 \pm 0.47)\end{array}$ & $\begin{array}{c}7.1-7.7 \\
(7.4 \pm 0.25) \\
\end{array}$ & $\begin{array}{c}0.30-0.50 \\
(0.40 \pm 0.11)\end{array}$ & $\begin{array}{c}0.05-0.09 \\
(0.08 \pm 0.02)\end{array}$ & $\begin{array}{c}0.05-0.09 \\
(0.07 \pm 0.01) \\
\end{array}$ \\
\hline & \multirow[t]{3}{*}{ August } & $\mathbf{F Z}$ & 0.70 & 26.30 & 4.60 & $\begin{array}{c}7.3-7.4 \\
(7.3 \pm 0.06)\end{array}$ & $\begin{array}{c}0.60-0.70 \\
(0.65 \pm 0.06)\end{array}$ & 0.05 & $\begin{array}{c}0.09-0.10 \\
(0.09 \pm 0.01)\end{array}$ \\
\hline & & TZ & $\begin{array}{c}9.8-30 \\
(18.9 \pm 8.76)\end{array}$ & $\begin{array}{c}26-26.2 \\
(26.0 \pm 0.10)\end{array}$ & $\begin{array}{c}3.5-4.6 \\
(4.1 \pm 0.47)\end{array}$ & $\begin{array}{c}7.6-7.9 \\
(7.8 \pm 0.17)\end{array}$ & $\begin{array}{c}0.30-0.80 \\
(0.57 \pm 0.17)\end{array}$ & $\begin{array}{c}0.05-0.09 \\
(0.07 \pm 0.02)\end{array}$ & $\begin{array}{c}0.04-0.09 \\
(0.07 \pm 0.02)\end{array}$ \\
\hline & & MZ & $\begin{array}{c}31-35 \\
(33.1 \pm 2.04)\end{array}$ & $\begin{array}{c}26.5-26.8 \\
(26.6 \pm 0.13)\end{array}$ & 4.50 & $\begin{array}{c}7.2-7.5 \\
(7.4 \pm 0.20) \\
\end{array}$ & $\begin{array}{c}0.40-1.20 \\
(0.77 \pm 0.40)\end{array}$ & $\begin{array}{c}0.07-0.09 \\
(0.08 \pm 0.01)\end{array}$ & 0.03 \\
\hline & \multirow[t]{3}{*}{ September } & $\mathbf{F Z}$ & 2.00 & 25.50 & 7.11 & 7.70 & 0.01 & 0.05 & 0.03 \\
\hline & & TZ & $\begin{array}{c}10.0-30 \\
(19.4 \pm 8.48)\end{array}$ & $\begin{array}{c}25.3-25.8 \\
(25.5 \pm 0.19)\end{array}$ & $\begin{array}{c}4.4-7.2 \\
(6.1 \pm 1.30) \\
\end{array}$ & $\begin{array}{c}7.8-8.0 \\
(7.9 \pm 0.08) \\
\end{array}$ & 0.01 & $\begin{array}{c}0.05-0.09 \\
(0.07 \pm 0.02)\end{array}$ & 0.03 \\
\hline & & MZ & $\begin{array}{c}31-35 \\
(33.3 \pm 1.86)\end{array}$ & 25.00 & $\begin{array}{c}7.2-7.4 \\
(7.3 \pm 0.09) \\
\end{array}$ & 8.00 & 0.01 & $\begin{array}{c}0.07-0.09 \\
(0.08 \pm 0.01) \\
\end{array}$ & $\begin{array}{c}0.09-0.10 \\
(0.09 \pm 0.01) \\
\end{array}$ \\
\hline \multirow[t]{6}{*}{ Dry } & October & $\mathbf{F Z}$ & $\begin{array}{c}2.3-2.4 \\
(2.3 \pm 0.06) \\
\end{array}$ & $\begin{array}{c}25.1-25.2 \\
(25.1 \pm 0.06)\end{array}$ & 8.00 & 8.00 & 0.40 & 0.05 & $\begin{array}{c}0.04-0.41 \\
(0.23 \pm 0.21) \\
\end{array}$ \\
\hline & & TZ & $\begin{array}{c}10.5-30.2 \\
(19.6 \pm 8.51)\end{array}$ & $\begin{array}{c}25.0-26.5 \\
(25.5 \pm 0.70)\end{array}$ & $\begin{array}{c}5.5-8.2 \\
(7.2 \pm 1.27) \\
\end{array}$ & $\begin{array}{c}7.6-8.0 \\
(7.9 \pm 0.18)\end{array}$ & $\begin{array}{c}0.04-0.60 \\
(0.36 \pm 0.24)\end{array}$ & $\begin{array}{c}0.05-0.09 \\
(0.07 \pm 0.02)\end{array}$ & $\begin{array}{c}0.04-0.28 \\
(0.15 \pm 0.11) \\
\end{array}$ \\
\hline & & MZ & $\begin{array}{c}32.1-35.3 \\
(33.7 \pm 1.68)\end{array}$ & $\begin{array}{c}26.1-26.7 \\
(25.3 \pm 0.23)\end{array}$ & $\begin{array}{c}6.8-7.1 \\
(6.9 \pm 1.15) \\
\end{array}$ & 8.00 & 0.40 & $\begin{array}{c}0.07-0.09 \\
(0.08 \pm 0.01)\end{array}$ & $\begin{array}{c}0.06-0.10 \\
(0.08 \pm 0.02) \\
\end{array}$ \\
\hline & \multirow[t]{3}{*}{ November } & $\mathbf{F Z}$ & $\begin{array}{c}4.4-4.5 \\
(4.4 \pm 0.06) \\
\end{array}$ & $\begin{array}{c}25.7-25.8 \\
(25.7 \pm 0.06)\end{array}$ & 7.40 & 8.00 & 1.00 & 0.07 & 0.03 \\
\hline & & $\mathbf{T Z}$ & $\begin{array}{c}11.2-30.8 \\
(20.7 \pm 8.48)\end{array}$ & $\begin{array}{c}22.6-25.6 \\
(24.3 \pm 1.29)\end{array}$ & $\begin{array}{c}7.5-7.9 \\
(7.6 \pm 0.19)\end{array}$ & $\begin{array}{c}7.7-8.0 \\
(7.9 \pm 0.12)\end{array}$ & $\begin{array}{c}0.04-0.90 \\
(0.39 \pm 0.41)\end{array}$ & $\begin{array}{c}0.05-0.09 \\
(0.07 \pm 0.02)\end{array}$ & $\begin{array}{c}0.03-0.04 \\
(0.03 \pm 0.01) \\
\end{array}$ \\
\hline & & MZ & $\begin{array}{c}34.0-36.1 \\
(35.0 \pm 1.10)\end{array}$ & $\begin{array}{c}26.0-27.6 \\
(26.7 \pm 0.82)\end{array}$ & $\begin{array}{c}7.6-7.7 \\
(7.6 \pm 0.05)\end{array}$ & $\begin{array}{c}7.5-7.8 \\
(7.6 \pm 0.15)\end{array}$ & $\begin{array}{c}0.80-0.90 \\
(0.87 \pm 0.05)\end{array}$ & $\begin{array}{c}0.06-0.09 \\
(0.08 \pm 0.01)\end{array}$ & $\begin{array}{c}0.03-0.06 \\
(0.04 \pm 0.01)\end{array}$ \\
\hline
\end{tabular}


Continued table 1.

\begin{tabular}{|c|c|c|c|c|c|c|c|c|c|}
\hline & December & $\mathbf{F Z}$ & 4.00 & $\begin{array}{c}25.9-26.0 \\
(25.9 \pm 0.06)\end{array}$ & 7.62 & 7.60 & 0.60 & 0.02 & 0.13 \\
\hline & & TZ & $\begin{array}{c}4.0-30.4 \\
(16.3 \pm 10.34)\end{array}$ & $\begin{array}{l}25.2-27.0 \\
(26.0 \pm 0.61)\end{array}$ & $\begin{array}{c}7.2-7.7 \\
(7.5 \pm 0.18)\end{array}$ & $\begin{array}{c}7.6-8.0 \\
(7.9 \pm 0.18)\end{array}$ & $\begin{array}{c}0.50-0.60 \\
(0.59 \pm 0.03)\end{array}$ & $\begin{array}{c}0.02-0.04 \\
(0.03 \pm 0.01)\end{array}$ & $\begin{array}{c}0.03-0.13 \\
(0.06 \pm 0.04)\end{array}$ \\
\hline & & MZ & $\begin{array}{c}32.4-35.7 \\
(34.0 \pm 1.74)\end{array}$ & $\begin{array}{c}25.4-25.6 \\
(25.5 \pm 0.08)\end{array}$ & 7.30 & $\begin{array}{c}7.4-7.5 \\
(7.4 \pm 0.03)\end{array}$ & $\begin{array}{c}0.60-0.70 \\
(0.64 \pm 0.05)\end{array}$ & $\begin{array}{c}0.04-0.07 \\
(0.05 \pm 0.01)\end{array}$ & $\begin{array}{c}0.03-0.04 \\
(0.03 \pm 0.01)\end{array}$ \\
\hline \multirow{3}{*}{\multicolumn{2}{|c|}{ January }} & $\mathbf{F Z}$ & 5.00 & $\begin{array}{c}25.1-25.2 \\
(25.1 \pm 0.06)\end{array}$ & 7.80 & 8.00 & $\begin{array}{c}0.69-0.70 \\
(0.69 \pm 0.01)\end{array}$ & 0.03 & 0.03 \\
\hline & & $\mathbf{T Z}$ & $\begin{array}{c}12.0-30.0 \\
(21.4 \pm 7.31)\end{array}$ & $\begin{array}{c}24.9-25.2 \\
(25.0 \pm 0.11)\end{array}$ & $\begin{array}{c}7.7-7.9 \\
(7.8 \pm 0.08)\end{array}$ & $\begin{array}{c}7.9-8.0 \\
(8.0 \pm 0.03)\end{array}$ & $\begin{array}{c}0.50-0.90 \\
(0.73 \pm 0.18)\end{array}$ & 0.02 & $\begin{array}{c}0.01-0.06 \\
(0.03 \pm 0.02)\end{array}$ \\
\hline & & MZ & $\begin{array}{c}34.0-36.5 \\
(35.2 \pm 1.35)\end{array}$ & $\begin{array}{c}23.0-25.7 \\
(24.0 \pm 1.14)\end{array}$ & $\begin{array}{c}7.9-8.1 \\
(8.0 \pm 0.10)\end{array}$ & $\begin{array}{c}7.2-7.4 \\
(7.3 \pm 0.08)\end{array}$ & $\begin{array}{c}0.80-0.81 \\
(0.81 \pm 0.01)\end{array}$ & 0.01 & $\begin{array}{c}0.03-0.10 \\
(0.06 \pm 0.04)\end{array}$ \\
\hline & February & $\mathbf{F Z}$ & 0.50 & $\begin{array}{c}26.4-26.6 \\
(26.5 \pm 0.10)\end{array}$ & $\begin{array}{c}7.4-7.5 \\
(7.4 \pm 0.01)\end{array}$ & $\begin{array}{c}7.2-7.3 \\
(7.3 \pm 0.01)\end{array}$ & $\begin{array}{c}0.60-0.61 \\
(0.61 \pm 0.01)\end{array}$ & 0.05 & 0.10 \\
\hline & & TZ & $\begin{array}{c}8.9-24.7 \\
(16.52 \pm 6.80)\end{array}$ & $\begin{array}{c}25.4-27.5 \\
(26.7 \pm 0.97)\end{array}$ & $\begin{array}{c}6.9-7.7 \\
(7.2 \pm 0.39)\end{array}$ & $\begin{array}{c}7.5-8.0 \\
(7.8 \pm 0.26)\end{array}$ & $\begin{array}{c}0.29-0.51 \\
(0.44 \pm 0.10)\end{array}$ & $\begin{array}{c}0.05-0.09 \\
(0.07 \pm 0.02)\end{array}$ & $\begin{array}{c}0.03-0.07 \\
(0.05 \pm 0.02)\end{array}$ \\
\hline & & MZ & $\begin{array}{c}29.5-33.5 \\
(31.5 \pm 2.13)\end{array}$ & $\begin{array}{c}26.5-27.1 \\
(26.7 \pm 0.27)\end{array}$ & 7.00 & $\begin{array}{c}7.9-8.0 \\
(7.9 \pm 0.06)\end{array}$ & $\begin{array}{c}0.80-0.90 \\
(0.85 \pm 0.05)\end{array}$ & $\begin{array}{c}0.07-0.09 \\
(0.08 \pm 0.01)\end{array}$ & $\begin{array}{c}0.05-0.09 \\
(0.07 \pm 0.02)\end{array}$ \\
\hline \multirow[t]{3}{*}{ Rainy } & March & $\mathbf{F Z}$ & 0.60 & $\begin{array}{c}24.5-24.6 \\
(24.5 \pm 0.06)\end{array}$ & 7.50 & 8.00 & 0.60 & 0.05 & $\begin{array}{c}0.06-0.07 \\
(0.06 \pm 0.01)\end{array}$ \\
\hline & & TZ & $\begin{array}{c}9.0-24.8 \\
(16.7 \pm 6.82)\end{array}$ & $\begin{array}{c}24.5-25.7 \\
(24.9 \pm 0.53)\end{array}$ & $\begin{array}{c}8.0-8.1 \\
(8.0 \pm 0.04) \\
\end{array}$ & 8.00 & $\begin{array}{c}0.39-0.50 \\
(0.43 \pm 0.05)\end{array}$ & $\begin{array}{c}0.05-0.09 \\
(0.07 \pm 0.02)\end{array}$ & $\begin{array}{c}0.05-0.07 \\
(0.06 \pm 0.01) \\
\end{array}$ \\
\hline & & MZ & $\begin{array}{c}29.6-33.6 \\
(31.6 \pm 2.14)\end{array}$ & $\begin{array}{c}25.6-25.8 \\
(25.6 \pm 0.08)\end{array}$ & $\begin{array}{c}7.5-8.0 \\
(7.8 \pm 0.24)\end{array}$ & 8.00 & $\begin{array}{c}0.50-0.61 \\
(0.55 \pm 0.06)\end{array}$ & $\begin{array}{c}0.07-0.09 \\
(0.08 \pm 0.01)\end{array}$ & $\begin{array}{c}0.03-0.20 \\
(0.11 \pm 0.09)\end{array}$ \\
\hline
\end{tabular}

In the freshwater zone, salinity was the variable that was most strongly correlated with the abundance of the species present $(\rho=-0.405)$, and in the transitional and marine zones, in addition to salinity ( $\rho=-0.380$ and -0.477 , respectively), the species abundance was correlated with the nitrate concentrations ( $\rho=0.389$ and 0.463 , respectively). Despite the variations recorded in the total phytoplankton abundance, with higher abundance in the rainy season $\left(8.36 \times 105 \pm 2.17 \times 10^{6}\right.$ cells $\left.L^{-1}\right)$ than in the dry season $\left(1.42 \times 10^{5} \pm 2.95 \times 10^{5}\right.$ cells $\left.L^{-1}\right)$, the variance partition analysis demonstrated a low explanation of the environmental variables for community abundance (Table 3 ). In the rainy season, environmental variables explained $41 \%$ of the variance in the freshwater zone (residuals $=0.59 \%$ ), $24 \%$ in the transition zone (residuals $=0.76 \%$ ), and $16 \%$ in the marine zone (residuals $=0.84 \%$ ). In the dry season, these factors explained $42 \%$ of the variance in the freshwater zone (residuals $=0.58 \%$ ), $14 \%$ in the transition zone (residuals $=0.86 \%$ ), and $17 \%$ in the marine zone (residuals $=0.83 \%)$. We observed that the environmental factor rain better explained community abundance in the rainy season and dissolved inorganic nutrients better explained abundance in the dry season (Table 3 ).

The NMDS analysis (stress $=0.16$ ) suggested that habitat differentiation was a predominant factor for community structure in the estuarine gradient. The analysis indicated a typical pattern of ordering of samples according to the composition of freshwater species in the freshwater zone, and the overlapping of samples from the transition and marine zones (Figure 4). The freshwater species Aulacoseira sp., A. granulata (Ehrenberg) Simonsen, Dictyosphaerium sp., Euglena gracilis, Geitlerinema sp., Phacus acutus Pochmann, $P$. longicauda (Ehrenberg) Dujardin and Scenedesmus acuminatus (Lagerheim) Chodat, predominated in the freshwater zone. The exception was a Scrippsiella cf. acuminata bloom in the freshwater zone, which is a typical marine species but had a wide distribution in the Paraguaçu estuarine gradient. Further, Nitzschia sp. and Peridinium sp., whose genera are found in marine and freshwater habitats also predominated in the freshwater zone. The maximum abundance of these species occurred during the rainy season. In the dry season, in addition to $S$. cf. acuminata species, 
Table 2. Phytoplankton community composition of the Paraguaçu estuarine gradient.

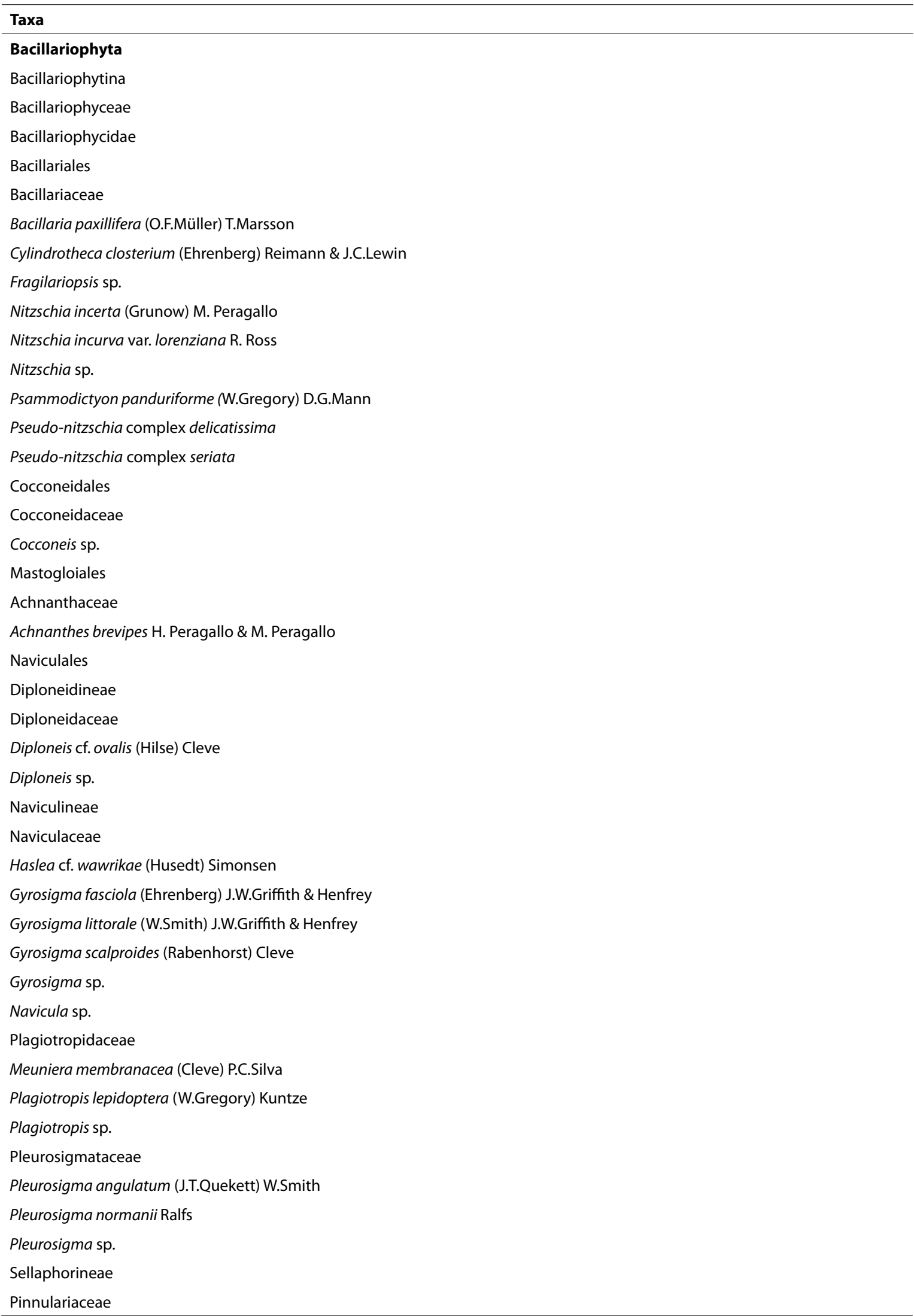




\section{Continued table 2.}

Pinnularia sp.

Surirellales

Entomoneidaceae

Entomoneis alata (Ehrenberg) Ehrenberg

Entomoneis gigantea (Grunow) Nizamuddin

Surirellaceae

Campylodiscus neofastuosus Ruck \& Nakov

Petrodictyon gemma (Ehrenberg) D.G.Mann

Thalassiophysales

Catenulaceae

Amphora sp.

Fragilariophycidae

Fragilariales

Fragilariaceae

Podocystis sp.

Licmophorales

Licmophoraceae

Licmophora sp.

Rhabdonematales

Grammatophoraceae

Grammatophora marina (Lyngbye) Kützing

Thalassionematales

Thalassionemataceae

Lioloma pacificum (Cupp) Hasle

Thalassionema frauenfeldii (Grunow) Tempère \& Peragallo

Thalassionema nitzschioides (Grunow) Mereschkowsky

Urneidophycidae

Rhaphoneidales

Asterionellopsidaceae

Asterionellopsis glacialis (Castracane) Round

Coscinodiscophytina

Coscinodiscophyceae

Aulacoseirales

Aulacoseiraceae

Aulacoseira granulata (Ehrenberg) Simonsen

Aulacoseira sp.

Rhizosoleniales

Rhizosoleniaceae

Dactyliosolen fragilissimus (Bergon) Hasle

Guinardia delicatula (Cleve) Hasle

Guinardia flaccida (Castracane) H.Peragallo

Guinardia striata (Stolterfoth) Hasle

Neocalyptrella robusta (G.Norman ex Ralfs) Hernández-Becerril \& Meave del Castillo

Rhizosolenia imbricata Brightwell 
Continued table 2.

Rhizosolenia setigera Brightwell

Triceratiales

Triceratiaceae

Triceratium favus Ehrenberg

Corethrophycidae

Corethrales

Corethraceae

Corethron criophilum Castracane

Coscinodiscophycidae

Coscinodiscales

Coscinodiscaceae

Coscinodiscus concinnus W. Smith

Coscinodiscus radiatus Ehrenberg

Coscinodiscus wailesii Gran \& Angst

Heliopeltaceae

Actinoptychus senarius (Ehrenberg) Ehrenberg

Hemidiscaceae

Hemidiscus cuneiformis Wallich

Melosirophycidae

Melosirales

Melosiraceae

Melosira sp.

Paraliophycidae

Paraliales

Paraliaceae

Paralia sulcata (Ehrenberg) Cleve

Mediophyceae

Biddulphiophycidae

Biddulphiales

Bellerocheaceae

Bellerochea

Bellerochea malleus (Brightwell) Van Heurck

Chaetocerotophycidae

Anaulales

Anaulaceae

Terpsinoë musica Ehrenberg

Chaetocerotales

Chaetocerotaceae

Bacteriastrum delicatulum Cleve

Chaetoceros aequatorialis Cleve

Chaetoceros affinis Lauder

Chaetoceros coarctatus Lauder

Chaetoceros costatus Pavillard 
Continued table 2.

Chaetoceros curvisetus Cleve

Chaetoceros danicus Cleve

Chaetoceros debilis Cleve

Chaetoceros didymus Ehrenberg

Chaetoceros lorenzianus Grunow

Chaetoceros peruvianus Brightwell

Chaetoceros radicans F.Schütt

Chaetoceros subtilis Cleve

Leptocylindraceae

Leptocylindrus danicus Cleve

Leptocylindrus minimus Gran

Hemiaulales

Hemiaulaceae

Cerataulina pelagica (Cleve) Hendey

Thalassiosirophycidae

Eupodiscales

Parodontellaceae

Trieres chinensis (Greville) Ashworth \& E.C.Theriot

Stephanodiscales

Stephanodiscaceae

Cyclotella meneghiniana Kützing

Cyclotella sp.

Thalassiosirales

Skeletonemataceae

Skeletonema cf. costatum (Greville) Cleve

Thalassiosiraceae

Thalassiosira cf. leptopus (Grunow) Hasle \& G.Fryxell

Thalassiosira subtilis (Ostenfeld) Gran

\section{Charophyta}

Zygnematophyceae

Zygnematophycidae

Desmidiales

Closteriaceae

Closterium leibleinii Kützing ex Ralfs

Closterium setaceum Ehrenberg ex Ralfs

Closterium sp.

Desmidiaceae

Cosmarium sp.

Staurastrum sp.

Zygnematales

Zygnemataceae

Spirogyra sp.

Chlorophyta

Chlorophytina 
Continued table 2.

\author{
Chlorophyceae \\ Chlamydomonadales \\ Volvocaceae \\ Pleodorina sp. \\ Sphaeropleales \\ Radiococcaceae \\ Gloeocystis sp. \\ Selenastraceae \\ Monoraphidium sp. \\ Scenedesmaceae \\ Tetradesmus lagerheimii M.J.Wynne \& Guiry \\ Coelastroideae \\ Coelastrum sp. \\ Desmodesmoideae \\ Desmodesmus communis (E.Hegewald) E.Hegewald \\ Trebouxiophyceae \\ Chlorellales \\ Chlorellaceae \\ Dictyosphaerium sp. \\ Cyanobacteria
}

Cyanophyceae

Nostocophycidae

Nostocales

Aphanizomenonaceae

Aphanizomenon sp.

Oscillatoriophycidae

Chroococcales

Chroococcaceae

Chroococcus sp.

Cyanothrichaceae

Johannesbaptistia sp.

Oscillatoriales

Coleofasciculaceae

Geitlerinema sp.

Microcoleaceae

Planktothrix isothrix (Skuja) Komárek \& Komárková

Planktothrix sp.

Trichodesmium erythraeum Ehrenberg ex Gomont

Oscillatoriaceae

Lyngbya confervoides C. Agardh ex Gomont

Lyngbya sp.

Spirulinales

Spirulinaceae

Spirulina sp. 
Continued table 2.

Synechococcophycidae

Synechococcales

Merismopediaceae

Merismopedia sp.

Pseudanabaenaceae

Pseudanabaena sp.

\section{Euglenozoa}

Dipilida

Euglenophyceae

Euglenophycidae

Euglenida

Euglenidae

Eugleninae

Euglena gracilis G.A.Klebs

Euglena sp.

Phacidae

Lepocinclis sp.

Phacus acuminatus A.Stokes

Phacus acutus Pochmann

Phacus longicauda (Ehrenberg) Dujardin

Eutreptiiida

Eutreptiidae

Eutreptiella sp.

Miozoa

Myzozoa

Dinozoa

Dinoflagellata

Dinophyceae

Dinophysales

Dinophysaceae

Metadinophysis sinensis Nie \& Wang

Gonyaulacales

Ceratiaceae

Tripos furca (Ehrenberg) F.Gómez

Tripos fusus (Ehrenberg) F.Gómez

Tripos hircus (Schröder) F.Gómez

Tripos muelleri Bory

Tripos trichoceros (Ehrenberg) Gómez

Gymnodiniales

Gymnodiniaceae

Akashiwo sanguinea (K.Hirasaka) Gert Hansen \& Moestrup

Margalefidinium polykrikoides (Margalef) F.Gómez, Richlen \& D.M.Anderson

Gyrodiniaceae

Gyrodinium sp. 
Continued table 2.

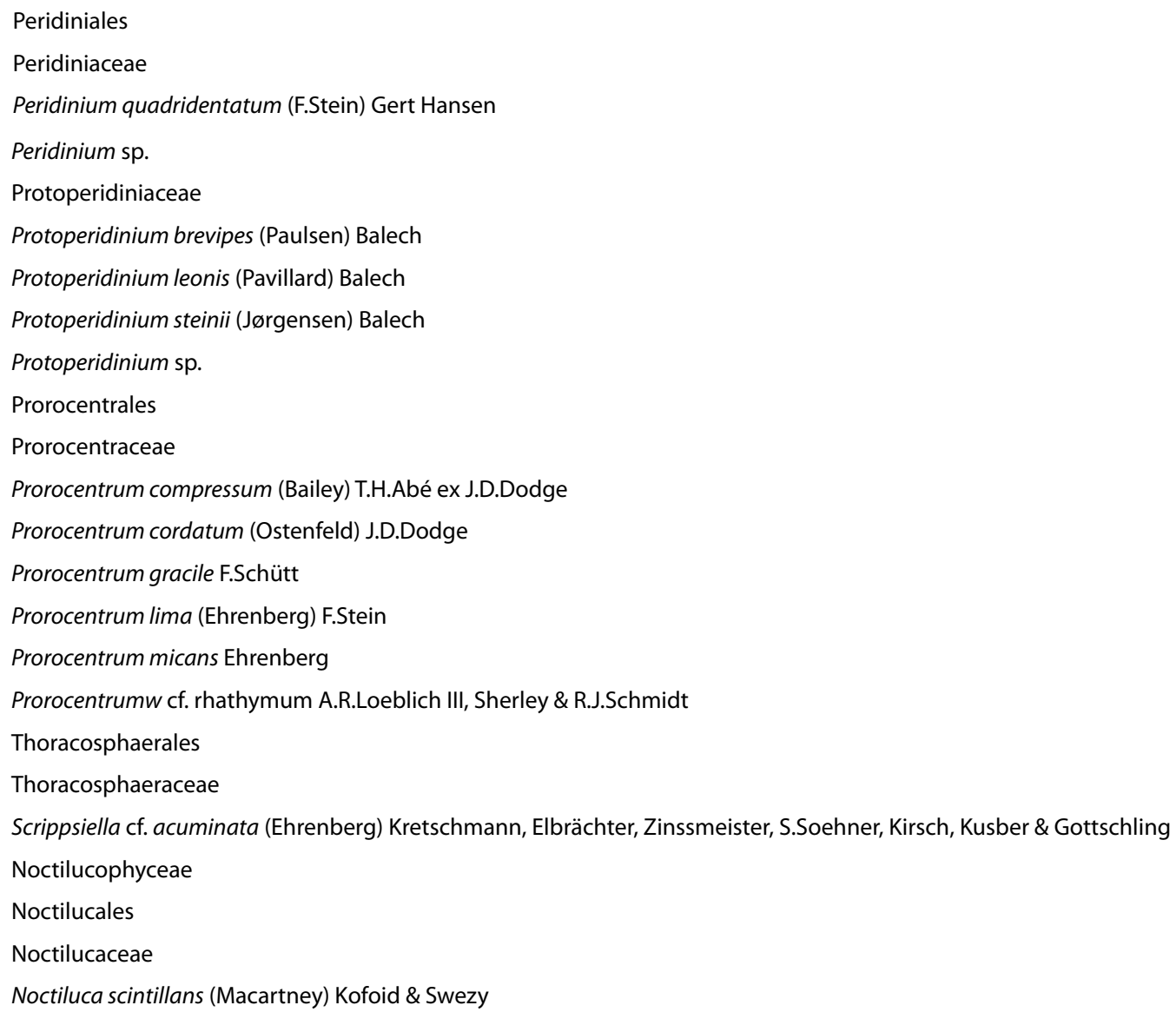

Dictyosphaerium sp. and Pleodorina sp., both freshwater species, predominated.

In the transitional zone, in addition to the species that formed blooms during the rainy season, the most abundant species were from marine habitats, with a predominance of diatoms, followed by dinoflagellates. In the dry season, besides the bloom of $A$. sanguinea, there was also a greater abundance of the dinoflagellate $S$. cf. acuminata. However, marine diatoms were predominant, with a greater abundance of the following species: Cerataulina pelagica (Cleve) Hendey, Cyclotella meneghiniana Kützing, Gyrosigma sp., Paralia sulcata (Ehrenberg) Cleve, Pseudo-nitzschia spp., Skeletonema cf. costatum, and Thalassiosira cf. leptopus (Grunow) Hasle \& G.Fryxell, besides, Euglena sp. and Eutreptiella sp., both Euglenophyceans. The marine zone presented a predominance of marine diatom species, together with the marine dinoflagellate Metadinophysis sinensis Nie \& Wang and the marine cyanobacteria Johannesbaptistia sp., in the rainy season. In the dry season, marine diatoms predominated: Cylindrotheca closterium (Ehrenberg) Reimann \& J.C.Lewin, Guinardia delicatula (Cleve) Hasle, Pseudo-nitzschia sp., and Leptocylindrus minimus Gran.

\section{DISCUSSION}

The historical average rainfall in the region of Paraguaçu River Estuary - Todos os Santos Bay is 2,100 $\mathrm{mm}$ per year, with the highest rainfall recorded between April and September, and a dry season from October to March (Cirano and Lessa, 2007; Hatje and Andrade, 2009). This pattern was verified throughout the study, with the exception of March, which presented greater precipitation $(161.15 \mathrm{~mm})$ than the typical average of the dry season $(44.33 \pm 31.68 \mathrm{~mm})$.

In general, during the rainy season, greater continental drainage influences water quality standards, increasing the concentration of dissolved inorganic nutrients in estuaries, for example, due to increased 


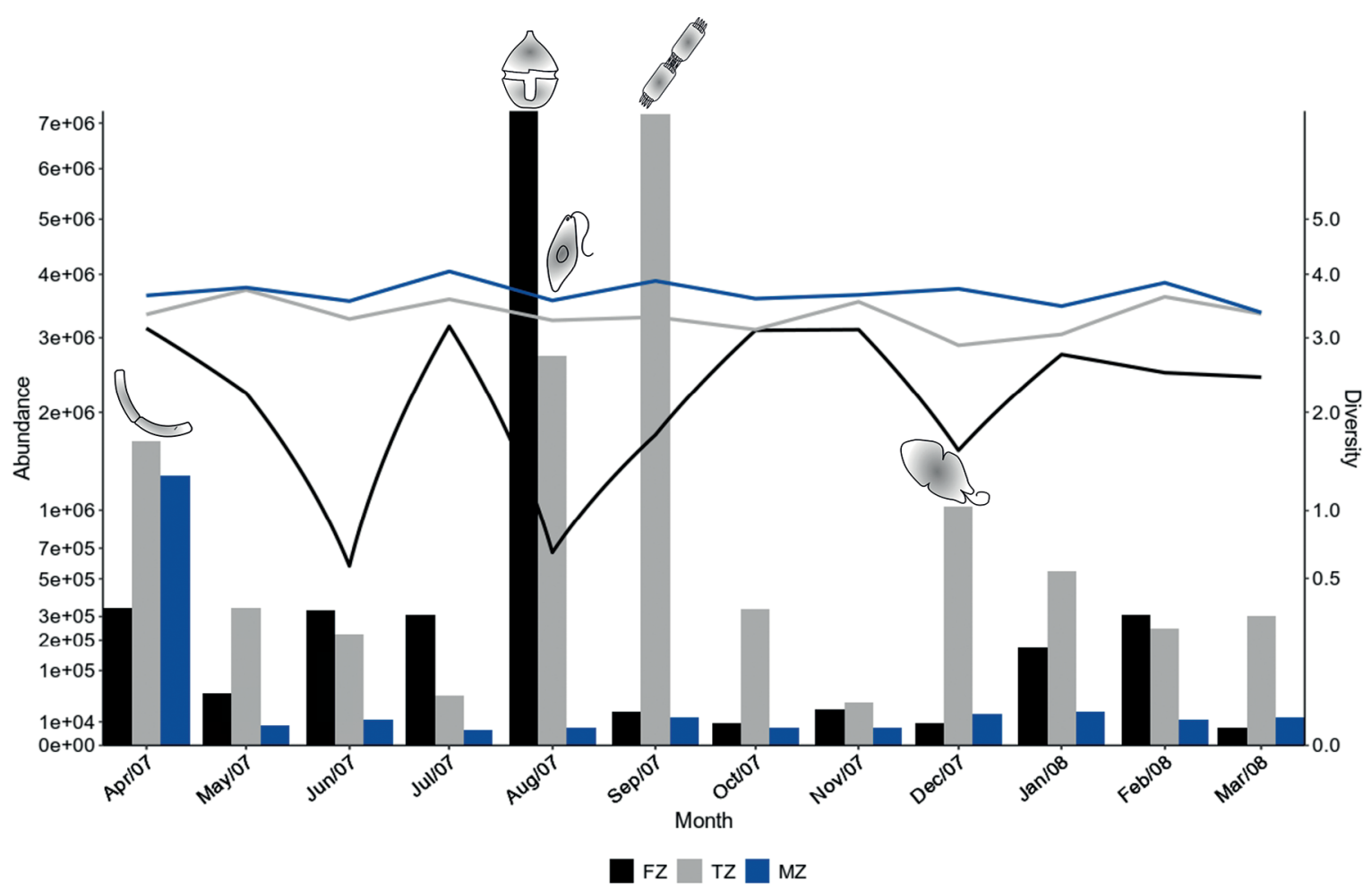

Figure 3. Average variation of the microphytoplankton abundance/cell density (cell L-1) (bars) and Shannon-Weaver Index $\left(\mathrm{H}^{\prime}\right)$ (lines) in the rainy (April-September, 2007, and March, 2008) and dry seasons (October-December, 2007, and January and February, 2008), and indication of the months of occurrence of the blooms of: Guinardia striata (TZ; April), Scrippsiella cf. acuminata (FZ; August), Euglena gracilis (TZ; August), Skeletonema cf. costatum (TZ; September) and Akashiwo sanguinea (TZ; December) in the Paraguaçu estuarine gradient. FZ = freshwater zone; $\mathrm{TZ}=$ transitional zone; $\mathrm{MZ}=$ marine zone.

Table 3. Relative contribution (\%) of environmental variables (physicochemical, nutrients and rain) to the microphytoplankton abundance in the rainy (April-September, 2007, and March, 2008) and dry (October-December, 2007, and January and February, 2008) seasons in the Paraguaçu estuarine gradient. $F Z$ = freshwater zone; $T Z$ = transitional zone; $\mathrm{MZ}=$ marine zone.

\begin{tabular}{|c|c|c|c|c|c|c|}
\hline \multirow[t]{2}{*}{ Variance partition variables } & \multicolumn{3}{|c|}{ Rainy } & \multicolumn{3}{|c|}{ Dry } \\
\hline & $\mathrm{FZ}$ & $\mathrm{TZ}$ & $M Z$ & $\mathrm{FZ}$ & $\mathrm{TZ}$ & $M Z$ \\
\hline Physicochemical & $16 \%$ & $19 \%$ & $11 \%$ & $18 \%$ & $4 \%$ & $11 \%$ \\
\hline Nutrients & $9 \%$ & $2 \%$ & $2 \%$ & $11 \%$ & $5 \%$ & $4 \%$ \\
\hline Rain & $6 \%$ & $2 \%$ & $2 \%$ & - & $1 \%$ & $1 \%$ \\
\hline Physicochemical + rain & $5 \%$ & - & $1 \%$ & - & - & - \\
\hline Nutrients + rain & $11 \%$ & $1 \%$ & - & $2 \%$ & $4 \%$ & - \\
\hline Physicochemical + nutrients & - & - & - & $11 \%$ & - & $1 \%$ \\
\hline
\end{tabular}

inputs from rivers (Noriega et al., 2019). In the case of the Paraguaçu River estuary, the influence of the Pedra do Cavalo dam that controls the flow of freshwater in the estuarine gradient must be considered, which ats as a physical barrier, retaining the material in the river channel. However, during our study period, the dam flow data was not available.
Thus, even with the variation recorded for the volume of rainfall throughout the study, discrete variations were recorded in the pattern of dissolved nutrients in the estuarine system, which maintained oligotrophic characteristics along the estuarine gradient, in the two pluviometric seasons. In the monitoring carried out between 2013 and 2014 in Todos 


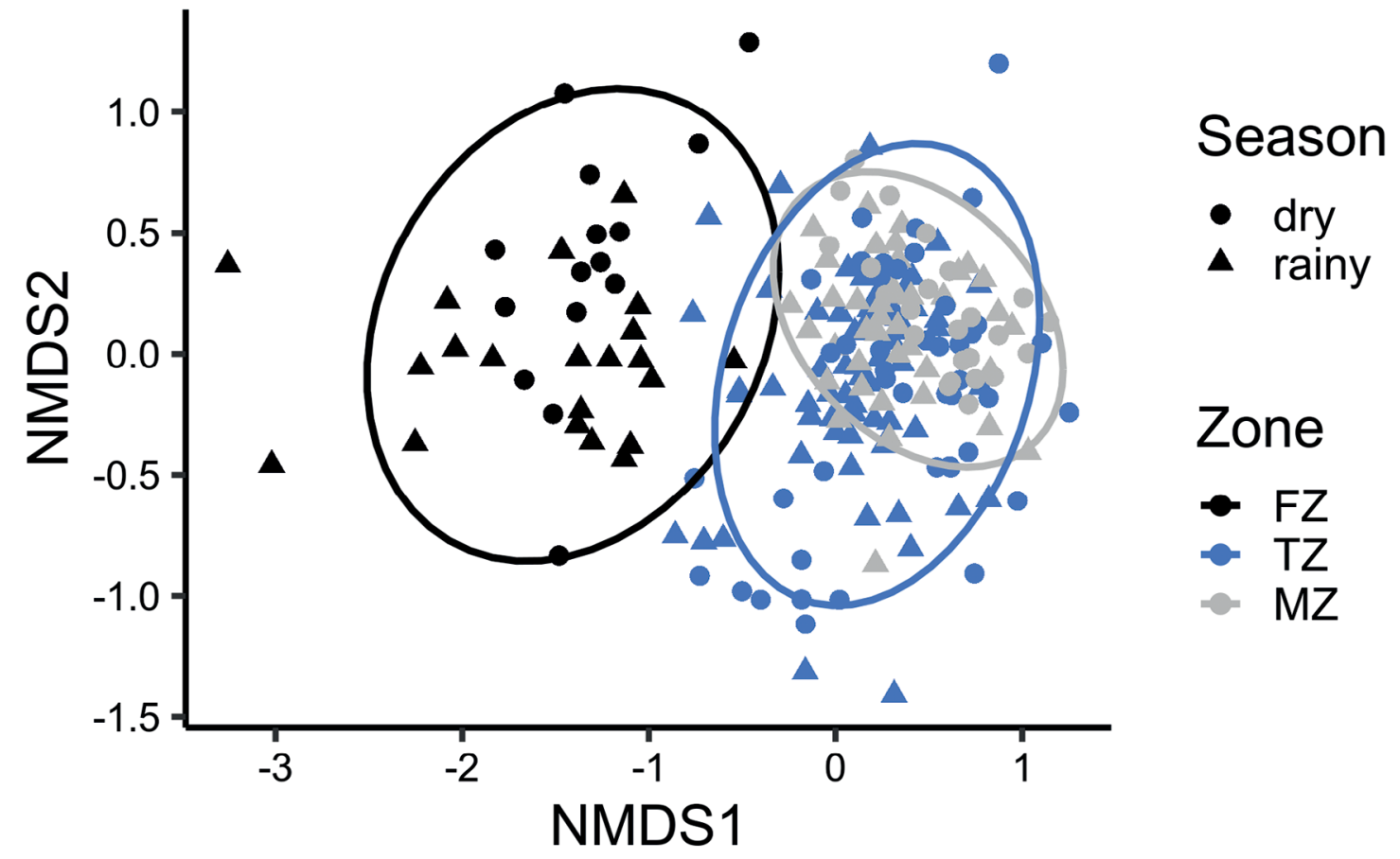

Figure 4. Non-metric multidimensional scaling (NMDS) analysis of the taxa identified in the three estuarine zones of the Paraguaçu estuarine gradient. $F Z$ = freshwater zone; $T Z$ = transitional zone; $M Z$ = marine zone.

os Santos Bay, with points distributed from the marine zone (coinciding with the delimitation that we used in the present study), an increase in the concentrations of dissolved nutrients during the period of greatest rainfall was registered. However, Lessa et al. (2018) identified dilution effects that maintained low nutrient concentrations in BTS, even under conditions of greater discharges into the Paraguaçu River.

We observed the typical species composition pattern from the tropical coastal environments (e.g. Rochelle-Newall et al., 2011; Chowdhury et al., 2017; Saifullah et al., 2019), with greater richness of the groups Charophyta, Chlorophyta and Euglenozoa in the zone under the most direct influence of the river input, and the greater richness of diatoms, followed by dinoflagellates, in the transition and marine zones. In the rainy season, species of Cyanophyta and Chlorophyta showed a greater spatial distribution in the estuarine gradient, certainly influenced by the greater river input in the system.

The algal blooms, in principle, represent a major source of food at the base of the aquatic food chain (Smayda, 1997). In this sense, the blooms recorded throughout the present study can be considered as a positive increase in the abundance and biomass of primary producers in the estuarine gradient, since they did not present any detectable harmful effects. However, it is important to consider that, among the five species that formed blooms, Skeletonema cf. costatum, Scrippsiella cf. acuminata, and Akashiwo sanguinea are classified as potentially harmful species (Castro and Moser, 2012), already registered as causing deleterious effects in some systems.

Skeletonema cf. costatum is a cosmopolitan, neritic species (Cupp, 1943; Hasle and Syvertsen, 1997), commonly recorded as forming summer blooms, under conditions of high temperatures, high irradiance, little mixing in the water column, higher concentrations of phosphate, and nitrate-limited (Shikata et al., 2008; Liu et al., 2005; Hu et al., 2011). We observed similar conditions: rainy season, very low concentrations of nitrite $(<0.01)$ and nitrate $(0.05 \mu \mathrm{M})$ and higher phosphate concentration $(0.13 \mu \mathrm{M})$ during the bloom of $S$. cf. costatum in the Paraguaçu estuarine system. Along the Brazilian coast, it has also been recorded at high densities in the Paranaguá 
Bay, influenced by the greater fluvial input, periods of high rainfall (Fernandes and Brandini, 2004) and associated with higher concentrations of ammonia in the water on the coast of Rio de Janeiro (Silva et al., 1988). This diatom is classified as a potentially harmful species since it can cause the death of fish and invertebrates when forming blooms with high biomass (e.g., Hallegraeff et al., 1995; Moestrup et al., 2009; Proença et al., 2011). This is therefore a potential risk, considering that one of the main ecosystem services of the estuarine system of the Paraguaçu River is the provision of fish for riverside families.

A prominent ecological characteristic of Scrippsiella cf. acuminata is its efficiency in forming temporary cysts, as a form of resistance, which can remain in benthic reservoirs and return to plankton, forming blooms under favorable conditions (Cloern and Dufford, 2005; Wang et al., 2007). These conditions generally include a high incidence of sunlight, high temperature, and high nutrient concentrations (Gárate-Lizárraga et al., 2009; Okolodkov et al., 2014; Kumar et al., 2018). However, even in an oligotrophic system such as the Paraguaçu River estuary, this species can be favored by small nutrient inputs in periods of higher rainfall. Records of blooms causing changes in water color and the death of fish and invertebrates, due to the depletion of dissolved oxygen, have been reported even under conditions of low nutrient availability (Hallegraef, 2003; Yin et al., 2008).

Guinardia striata and Euglena gracilis are species that are considered non-harmful, the first a marine species and the second, a species typical of freshwater. These species are often reported as forming blooms in their respective habitats. Although Whichard et al. (2008) point to possible deleterious effects of $G$. striata, leading to a reduction in the incubation success of eggs and an increase in the incidence of larval deformity of copepods, no ecological impacts were registered due to blooms of this species in the coastal waters of Cabo Frio - Rio de Janeiro, Ubatuba - São Paulo, and the Paranaguá Bay - Paraná. These are sites associated with conditions of high temperature, low nutrient concentration, and increased rainfall (Silva et al., 1988; Sassi and Kutner, 1982; Fernandes and Brandini, 2004), conditions similar to those recorded during the bloom period of the species in the Paraguaçu estuary.
The Euglena gracilis bloom is notable because this is an indicator species of organic pollutants (Li et al., 2014). Although we have shown oligotrophic characteristics in the estuarine gradient, it must be considered that the entire region surrounding the estuary and BTS has problems with basic sanitation infrastructure, with domestic sewage discharging into the water bodies. Further, an additional potential risk of water contamination could be due to the industrial activities developed in BTS (Hatje and Andrade, 2009).

Harmful blooms of Akashiwo sanguinea can mainly cause conditions of anoxia in the water column, or the clogging of gills of fish and invertebrates (Hallegraeff, 2003; Jessup et al., 2009). In BTS, the bloom of this species, which motivated the present study, was favored by conditions such as low wind intensity and high temperature, after a period of heavy rains in the region of the head of the river, which caused a high intake of freshwater into the estuarine system. This is evident from the reduction of salinity to below 30, whereas the normal range varies between 32 and 39 (Lessa et al., 2018). Increased rainfall can result in the opening of the gates of the Pedra do Cavalo dam. This process promotes greater freshwater fluxes and consequently large dissolved nutrients input, which are carried along the estuary up to BTS.

It is suggested that the establishment of these favorable environmental conditions provided the breakdown of resistance cysts deposited in the system, thus forming an enormous bloom of $A$. sanguinea, as evidenced by Badylak et al. (2017) in the Caloosahatchee estuary in Florida. According to the authors, the deposit of cysts subsidized the formation of a bloom, under conditions of low salinity, high temperature, and increase in the total concentrations of phosphorus and nitrogen. In estuaries subject to large freshwater inflows, $A$. sanguinea presents cell densities around $7.4 \times 10^{5}$ cells $\mathrm{L}^{-1}$, in a great range of salinities (Badylak et al., 2014), forming blooms (1.5 $x 10^{6}$ cells $\left.\mathrm{L}^{-1}\right)$ in conditions of warm surface waters and near the end of the upwelling phase (White et al., 2014). This species has a wide distribution in the estuarine systems of Northeast Brazil, presenting shortterm variations in abundance under tidal effects (e.g. Affe et al., 2018; Santos et al., 2020). Besides, its density increases during the rainy seasons, in low tide 
periods, especially under conditions of increasing concentrations of organic and inorganic phosphorus (Koening et al., 2014).

Although blooms did not cause any notable changes in the water column, it is important to consider that a possible establishment of favorable environmental conditions can provide the formation of a more expressive bloom, which can trigger harmful effects on the system. The existence of the anthropogenic pressures and the occurrence of potentially harmful species need to be considered to better understand the dynamics of the phytoplankton community in the estuarine system, as well as to guide initiatives to mitigate possible new harmful algal blooms.

\section{ACKNOWLEDGMENTS}

LPC acknowledges Coordenação de Aperfeiçoamento de Pessoal de Nível Superior Brasil (CAPES-Finance Code 001) for a Doctoral Scholarship. JMCN acknowledges Conselho Nacional de Desenvolvimento Científico e Tecnológico (CNPq \# 308542/2018-5) for a Productivity Scholarship in Research.

\section{AUTHOR CONTRIBUTIONS}

H.M.J.A., L.A.O.P. and J.M.C.N.: contributed to the concept and design of the study.

H.M.J.A. and D.S.B.R.: conceived the statistical analyzes. H.M.J.A.: wrote original draft.

H.M.J.A., L.P.C. and D.S.B.R.: wrote review \& editing. All authors read and approved the final version of the article.

\section{REFERENCES}

AFFE, H. M. J, CAIRES, T. A., SILVA, E. M. \& NUNES, J. M. C. 2016. Floración de Trichodesmium erythraeum en la región costera tropical de Brasil. Revista de Biología Marina y Oceanografía, 51(1), 175-179, DOI: https://doi.org/10.4067/S071819572016000100017

AFFE, H. M. J., MENEZES, M. \& NUNES, J. M. C. 2018. Microphytoplankton in a tropical oligotrophic estuarine system: spatial variations and tidal cycles. Revista Brasileira de Botânica, 41, 1-13.

BADYLAK, S., PHLIPS, E. J. \& MATHEWS, A. L. 2014. Akashiwo sanguinea (Dinophyceae) blooms in a sub-tropical estuary: an alga for all seasons. Plankton and Benthos Research, 9(3), 147-55, DOI: https://doi.org/10.3800/pbr.9.147
BADYLAK, S., PHLIPS, E. J., MATHEWS, A. L. \& KELLEY, K. 2017. In situ observations of Akashiwo sanguinea (Dinophyceae) displaying life cycle stages during blooms in a subtropical estuary. Botanica Marina, 60(6), 653-664.

BORCARD, D., LEGENDRE, P. \& DRAPEAU, P. 1992. Partialling out the spatial component of ecological variation. Ecology, 73(3), 1045-1055.

CASTRO, N. O. \& MOSER, G. A. O. 2012. Florações de algas nocivas e seus efeitos ambientais. Oecologia Australis, 16(2), 235-264.

CHOWDHURY, M., HARDIKAR, R., KESAVAN, H. C., THOMAS, J., MITRA, A., ROKADE, M. A., NAIDU, V. S. \& SUKUMARAN, S. 2017. Nutrient stoichiometry and freshwater flow in shaping of phytoplankton population in a tropical monsoonal estuary (Kundalika Estuary). Estuarine, Coastal and Shelf Science, 198(Pt A), 73-91, DOI: https://doi. org/10.1016/j.ecss.2017.08.019

CIRANO, M. \& LESSA, G. C. 2007. Oceanographic characteristics of Baía de Todos os Santos, Brazil. Revista Brasileira de Geofísica, 25(4), 363-387.

CLOERN, J. E. \& DUFFORD, R. 2005. Phytoplankton community ecology: principles applied in San Francisco Bay. Marine Ecology Progress Series, 285, 11-28.

CÓRDOBA-MENA, N., FLOREZ-LEIVA, L., ATEHORTÚA, L. \& OBANDO, E. 2020. Changes in phytoplankton communities in a tropical estuary in the Colombian Caribbean Sea. Estuaries and Coasts, 43, 2106-27, DOI: https://doi.org/10.1007/s12237-020-00750-z

CRA (Conselho Regional de Administração da Bahia). 2005. Análise preliminar de risco à saúde humana. Relatório Síntese. Bahia: Consórcio BTS Hydros CH2MHILL. Available at: http://www.ceama.mpba.mp.br/biblioteca-virtual-nbts/ biblioteca-digital-1103/doc_view/2044-analise-preliminar-derisco-a-saude-humana-relatorio-sintese.html (accessed in 26/ Mar/2021)

CUPP, E. E. 1943. Marine plankton diatoms of the west coast of North America. Berkeley: University of California Press.

EGERTON, T. A., MORSE, R. E., MARSHALL, H. G. \& MULHOLLAND, M. R. 2014. Emergence of algal blooms: the effects of short-term variability in water quality on phytoplankton abundance, diversity, and community composition in a tidal estuary. Microorganisms, 2(1), 33-57, DOI: https://doi. org/10.3390/microorganisms2010033

FERNANDES, L. F. \& BRANDINI, F. P. 2004. Diatom associations in shelf waters off Parana State, Southern Brazil: annual variation in relation to environmental factors. Brazilian Journal of Oceanography, 52(1), 19-34.

GÁRATE-LIZÁRRAGA, I., BAND-SCHMIDT, C. J., LÓPEZ-CORTÉS, D. J. \& MUÑETÓN-GÓMEZ, M. D. S. 2009. Bloom of Scrippsiella trochoidea (Gonyaulacaceae) in a shrimp pond in the southwestern Gulf of California, Mexico. Marine Pollution Bulletin, 58(1), 145-149.

GENZ, F., LESSA, G. C. \& CIRANO, M. 2008. Vazão mínima para estuários: um estudo de caso no rio Paraguaçu (BA). Revista Brasileira de Recursos Hídricos, 13(3), 73-82.

HALLEGRAEFF, G. M., ANDERSON, D. M. \& CEMBELLA, A. D. 1995. Manual on harmful marine microalgae. IOC Manuals and Guides $N^{\circ} 33$. Paris: UNESCO Publishing.

HALLEGRAEFF, G. M. 2003. Harmful algal blooms: a global overview. Manual on harmful marine microalgae, 33, 1-22. 
HALLEGRAEFF, G. M. 2010. Ocean climate change, phytoplankton community responses, and harmful algal blooms: a formidable predictive challenge. Journal of Phycology, 46(2), 220-235, DOI: http://dx.doi.org/10.1111/j.1529-8817.2010.00815.x

HASLE, G. R. \& SYVERTSEN, E. E. 1997. Dinoflagellates. In: TOMAS, C. R. (ed.). Identifying marine phytoplankton. San Diego: Academic Press, pp. 387-584.

HATJE, V. \& ANDRADE, J. B. 2009. Baía de Todos os Santos: aspectos oceanográficos. Salvador: EDUFBA.

HEISLER, J., GLIBERT, P. M., BURKHOLDER, J. M., ANDERSON, D. M., COCHLAN, W., DENNISON, W. C., DORTCH, Q., GOBLER, C. J., HEIL, C. C., HUMPHRIES, E., LEWITUS, A., MAGNIEN, R., MARSHALL, H. G., SELLNER, K., STOCKWELL, D. A., STOECKER, D. K. \& SUDDLESON, M. 2008. Eutrophication and harmful algal blooms: a scientific consensus. Harmful Algae, 8(1), 3-13, DOI: http://dx.doi.org/10.1016/j.hal.2008.08.006

HU, H. H., ZHANG, J. \& CHEN, W. D. 2011. Competition of bloomforming marine phytoplankton at low nutrient concentrations. Journal of Environmental Sciences, 23(4), 656-663.

JAKOBSEN, H. H., BLANDA, E., STAEHR, P. A., HØJGÅRD, J. K., RAYNER, T. A., PEDERSEN, M. F., JEPSEN P. M. \& HANSEN, B. W. 2015. Development of phytoplankton communities: implications of nutrient injections on phytoplankton composition, $\mathrm{pH}$ and ecosystem production. Journal of Experimental Marine Biology and Ecology, 473, 81-89, DOI: https://doi. org/10.1016/j.jembe.2015.08.011

JESSUP, D. A., MILLER, M. A., RYAN, J. P., NEVINS, H. M., KERKERING, H. A., MEKEBRI, A., CRANE, D. B., JONHSON, T. A. \& KUDELA, R. M. 2009. Mass stranding of marine birds caused by a surfactant-producing red tide. PLoS One, 4(2), e4550.

KOENING, M. L., FLORES MONTES, M. J., ESKINAZI LEÇA, E. \& TIBURCIO, A. S. X. S. 2014. New record of Akashiwo sanguinea (Dinophyta) in the tropical estuarine waters of Northeastern Brazil (Western Atlantic). Brazilian Journal of Biology, 74(1), 191-198.

KUMAR, P. S., KUMARASWAMI, M., RAO, G. D., EZHILARASAN, P., SIVASANKAR, R., RAO, V. R. \& RAMU, K. 2018. Influence of nutrient fluxes on phytoplankton community and harmful algal blooms along the coastal waters of southeastern Arabian Sea. Continental Shelf Research, 161, 20-28.

LESSA, G. C., CIRANO, M., GENZ, F., TANAJURA, C. A. S. O. \& SILVA R. R. 2009. Oceanografia Física. In: HATJE, V. \& ANDRADE, J. B. (orgs.). Baía de Todos os Santos: aspectos oceanográficos. Salvador: EDUFBA.

LESSA, G. C., DOMINGUEZ, J. M. L., BITTENCOURT, A. C. S. P. \& BRICHTA, A. 2001. The tide and tidal circulation of Todos os Santos Bay, Northeast Brazil: a general characterization. Anais da Academia Brasileira de Ciências, 73(2), 245-261.

LESSA, G. C., SOUZA, M. F. L., MAFALDA JUNIOR, P. O., GOMES, D. F., SOUZA, C. S., TEIXEIRA, C. E. P., SOUZA, J. R. L. B. \& ZUCCHI, M. R. 2018. Variabilidade intra-anual da oceanografia da Baía de Todos os Santos: evidências de três anos de monitoramento. In: HATJE, V., DANTAS, L. M. V. \& ANDRADE, J. B. (orgs.). Baía de Todos os Santos: avanços nos estudos de longo prazo. Salvador: EDUFBA.

LI, M., GAO, X., WU, B., QIAN, X., GIESY, J. P. \& CUI, Y. 2014. Microalga Euglena as a bioindicator for testing genotoxic potentials of organic pollutants in Taihu Lake, China. Ecotoxicology, 23(4), 633-640.
LITCHMAN, E. \& KLAUSMEIER, C. A. 2008. Trait-based community ecology of phytoplankton. Annual Review of Ecology, Evolution, and Systematics, 39, 615-639.

LIU, D. Y., SUN, J., ZOU, J. Z. \& ZHANG, J. 2005. Phytoplankton succession during a red tide of Skeletonema costatum in Jiaozhou Bay of China. Marine Pollution Bulletin, 50(1), 91-94.

MOESTRUP, Ø., AKSELMAN-CARDELLA, R., CRONBERG, G., ELBRAECHTER, M., FRAGA, S., HALIM, Y., HANSEN, G., HOPPENRATH, M., LARSEN, J., LUNDHOLM, N., NGUYEN, L. N. \& ZINGONE, A. 2009. IOC-UNESCO taxonomic reference list of harmful microalgae [online]. Paris: UNESCO. Avaliable at: http://www.marinespecies.org/HAB [Acessed 22 Apr 2020].

MOORE, S. K., TRAINER, V. L., MANTUA, N. J., PARKER, M. S., LAWS, E., BACKER, L. C. \& FLEMING, L. E. 2008. Impacts of climate variability and future climate change on harmful algal blooms and human health. Environmental Health, 7(Suppl 2), S4, DOI: http://dx.doi.org/10.1186/1476-069X-7-S2-S4

MORSE, R. E., MULHOLLAND, M. R., EGERTON, T. A. \& MARSHALL, H. G. 2014. Daily variability in phytoplankton abundance and nutrient concentrations in a tidally dominated eutrophic estuary. Marine Ecology Progress Series, 503, 59-74.

MOSER, G. A. O., PIEDRAS, F. R., OAQUIM, A. B. J., SOUZA, D. S., LELES, S. G., DE LIMA, D. T. \& FERNANDES, A. M. 2017. Tidal effects on phytoplankton assemblages in a near-pristine estuary: a trait-based approach for the case of a shallow tropical ecosystem in Brazil. Marine Ecology, 38(4), e12450, DOl: https://doi.org/10.1111/maec.12450

NORIEGA, C., ARAUJO, M., FLORES-MONTES, M. \& ARAUJO, J. 2019. Trophic dynamics (Dissolved Inorganic Nitrogen-DIN and Dissolved Inorganic Phosphorus-DIP) in tropical urban estuarine systems during periods of high and low river discharge rates. Anais da Academia Brasileira de Ciências, 91(2), e20180244, DOI: https://doi.org/10.1590/0001-3765201920180244

OKOLODKOV, Y. B., MERINO-VIRGILIO, F. D. C., AGUILAR-TRUJILLO, A. C., OSORIO-MORENO, I. \& HERRERA-SILVEIRA, J. A. 2014. The genus Scrippsiella (Dinoflagellata) in coastal waters of the northern Yucatan Peninsula, SE Gulf of Mexico. Boletín de la Sociedad Mexicana de Ficología y de la Sociedad Ficológica de América Latina y el Caribe, 4, 21-32.

OKSANEN, J., BLANCHET, F. G., FRIENDLY, M., KINDT, R., LEGENDRE, P., MCGLINN, D., MINCHIN, P. R., O'HARA, R. B., SIMPSON, G. L., SOLYMOS, P., HENRY, M., STEVENS, H., SZOECS, E. \& WAGNER, H. 2019. Vegan: community ecology package - 2018. $R$ package version, 2, 17-4.

PERES-NETO, P. R., LEGENDRE, P., DRAY, S. \& BORCARD, D. 2006. Variation partitioning of species data matrices: estimation and comparison of fractions. Ecology, 87(10), 2614-2625.

PROENÇA, L. A. O., FONSECA, R. S. \& PINTO, T. O. 2011. Microalgas em área de cultivo do litoral de Santa Catarina. São Carlos: RiMa.

R CORE TEAM. 2020. $R$ : a language and environment for statistical computing. Vienna: R Foundation for Statistical Computing.

RABALAIS, N. N. 2002. Nitrogen in aquatic ecosystems. Ambio, $31(2), 102-112$.

REIS-FILHO, J. A., SILVA, E. M., NUNES, J. A. C. C. \& BARROS, F. 2012. Effects of a red tide on the structure of estuarine fish assemblages in northeastern Brazil. International Review of Hydrobiology, 97(5), 389-404, DOI: https://doi.org/10.1002/ iroh.201101457 
ROCHELLE-NEWALL, E. J., CHU, V. T., PRINGAULT, O., AMOUROUX, D., ARFI, R., BETTAREL, Y., BOUVIER, T., BOUVIER, C., GOT, P., NGUYEN, T. M. H., MARI, X., NAVARRO, P., DUONG, T. N., CAO, T. T. T., PHAM, T. T., OUILLON, S. \& TORRÉTON, J. P. 2011. Phytoplankton distribution and productivity in a highly turbid, tropical coastal system (Bach Dang Estuary, Vietnam). Marine Pollution Bulletin, 62(11), 2317-2329.

SAIFULLAH, A. S. M., KAMAL, A. H. M., IDRIS, M. H. \& RAJAEE, A. H. 2019. Community composition and diversity of phytoplankton in relation to environmental variables and seasonality in a tropical mangrove estuary. Regional Studies in Marine Science, 32, e100826, DOI: https://doi.org/10.1016/j. rsma.2019.100826

SANTANA, R., TEIXEIRA, C. \& LESSA, G. 2018. The impact of different forcing agents on the residual circulation in a tropical estuary (Baía de Todos os Santos, Brazil). Journal of Coastal Research, 34(3), 544-558, DOI: https://doi.org/10.2112/ JCOASTRES-D-17-00044.1

SANTOS, L. P. N, AFFE, H. M. J. \& NUNES, J. M. C. 2020. Microfitoplâncton na Baía de Todos Os Santos (Brasil): composição, diversidade e abundância em um curto período de tempo. Acta Botanica Malacitana, 45, 27-36, DOI: https://doi. org/10.24310/abm.v45i.5301

SASSI, R. \& KUTNER, M. B. 1982. Variação sazonal do fitoplâncton da região do Saco da Ribeira (Lat. 2330'S; Long. 4507'W), Ubatuba, Brasil. Boletim do Instituto Oceanográfico, 31(2), 29-42.

SHIKATA, T., NAGASOE, S., MATSUBARA, T., YOSHIKAWA, S., YAMASAKI, Y., SHIMASAKI, Y., OSHIMA, Y., JENKINSON, I. R. \& HONJO, T. 2008. Factors influencing the initiation of blooms of the raphidophyte Heterosigma akashiwo and the diatom Skeletonema costatum in a port in Japan. Limnology and Oceanography, 53(6), 2503-2518.
SILVA, N. M., VALENTIN, J. L. \& BASTOS, C. T. B. 1988. O microfitoplâncton das águas costeiras do litoral fluminense (Estado do Rio de Janeiro): lista de espécies e aspectos ecológicos. Boletim do Instituto Oceanográfico, 36(1-2), 01-16.

SMAYDA, T. J. 1997. What is a bloom? A commentary. Limnology and Oceanography, 42(5 Pt 2), 1132-1136, DOI: https://doi. org/10.4319/lo.1997.42.5_part_2.1132

STRICKLAND, J. D. H. \& PARSONS, T. R. 1972. A practical handbook of seawater analysis. Ottawa: Fisheries Research Board of Canada.

UTERMÖHL, H. 1958. Zur Vervollkommnung der quantitativen Phytoplankton-Methodik. International Association of Theoretical and Applied Limnology, 9, 1-38, DOI: https://doi.org/1 0.1080/05384680.1958.11904091

WANG, Z. H., QI, Y. Z. \& YANG, Y. F. 2007. Cyst formation: an important mechanism for the termination of Scrippsiella trochoidea (Dinophyceae) bloom. Journal of Plankton Research, 29(2), 209-218.

WHITE, A. E., WATKINS-BRANDT, K. S., MCKIBBEN, S. M., WOOD, A. M., HUNTER, M., FOSTER, Z., DU, X. \& PETERSON, W. T. 2014. Large-scale bloom of Akashiwo sanguinea in the Northern California current system in 2009. Harmful Algae, 37, 38-46, DOI: https://doi.org/10.1016/j.hal.2014.05.004

WICHARD, T., POULET, S. A., BOULESTEIX, A. L., LEDOUX, J. B., LEBRETON, B., MARCHETTI, J. \& POHNERT, G. 2008. Influence of diatoms on copepod reproduction. II. Uncorrelated effects of diatom-derived $a, \beta, \gamma, \delta$-unsaturated aldehydes and polyunsaturated fatty acids on Calanus helgolandicus in the field. Progress in Oceanography, 77(1), 30-44.

YIN, K., SONG, X. X., LIU, S., KAN, J. \& QIAN, P. Y. 2008. Is inorganic nutrient enrichment a driving force for the formation of red tides? A case study of the dinoflagellate Scrippsiella trochoidea in an embayment. Harmful Algae, 8, 54-59. 